\title{
CIH: from sleep apnea to breath-hold diving
}

\author{
Is sympathetic activation inevitable?
}

\author{
Mark W. Chapleau
}

Published online: 20 April 2010

(C) Springer-Verlag 2010

Recurrent apneas associated with sleep-disordered breathing increase sympathetic nerve activity and arterial blood pressure, and enhance sympathetic and ventilatory responses to acute hypoxia [14, 19]. Chronic intermittent hypoxia $(\mathrm{CIH})$ and activation of arterial carotid body chemoreceptors are primarily responsible for driving the increase in sympathetic activity [4, 17]. The excessive sympathetic activity and hypertension are sustained and contribute to high morbidity and mortality in afflicted patients [15, 19].

Certain recreational and vocational activities involve episodes of volitional apnea. One example is underwater breath-hold diving. In this issue of Clinical Autonomic Research, Breskovic and colleagues [1] address the question: Do elite breath-hold divers exhibit exaggerated increases in muscle sympathetic nerve activity (MSNA) and ventilation during exposure to acute hypoxia? Such a finding would raise concern that the $\mathrm{CIH}$ imposed by these types of activities may increase cardiovascular risk in otherwise healthy individuals.

The investigators measured arterial oxygen saturation, ventilation, MSNA, blood pressure, heart rate and stroke volume in elite divers and healthy control subjects at

M. W. Chapleau $(\bowtie)$

Department of Internal Medicine, Cardiovascular Center,

The University of Iowa Carver College of Medicine,

200 Hawkins Drive, Iowa City, IA 52242, USA

e-mail: mark-chapleau@uiowa.edu

M. W. Chapleau

Department of Molecular Physiology and Biophysics, Cardiovascular Center, The University of Iowa Carver College

of Medicine, 200 Hawkins Drive, Iowa City, IA 52242, USA

M. W. Chapleau

Veterans Affairs Medical Center, Iowa City, IA 52246, USA baseline (while breathing room air) and during progressive isocapnic hypoxia [1]. Resting blood pressure and MSNA were not significantly different in divers versus control subjects. Furthermore, hypoxia-induced increases in ventilation and MSNA were also not different between the groups. While good news for the divers, these negative results raise several important questions.

\section{Is the degree of CIH in divers sufficient to induce sustained sympathetic activation?}

The frequency of apneas in elite breath-hold divers, at least during the diving season, has been reported to approach that seen in patients with sleep apnea [1-3, 7]. Furthermore, while a single maximum end-inspiratory breath-hold has little effect on arterial oxygenation in control subjects, the longer maximum breath-hold times in elite divers (up to $6 \mathrm{~min}$, world record $=\sim 10 \mathrm{~min}$ ) are associated with marked arterial oxygen desaturation and pronounced increases in MSNA and blood pressure [5, 7]. The chemoreceptor reflex is surely activated when elite divers breath-hold. Thus, it appears likely that the frequency of apneas and degree of hypoxemia were sufficient to induce sympathoexcitation and hypertension in the elite divers in this study. Nonetheless, the present study is limited by the lack of information on the frequency of near-maximum apneas experienced over time by the elite divers.

Of greater significance is the 1-2 month delay between intense apnea training and the day of the study [1]. CIHinduced increases in chemoreceptor sensitivity and sympathetic activity in animal models can be reversed as soon as 10 days after terminating the $\mathrm{CIH} \mathrm{[17].} \mathrm{Effects} \mathrm{of} \mathrm{CIH}$ may have dissipated before the divers were studied. On the other hand, the same group of investigators have reported 
in a separate study that resting MSNA and blood pressure, and responses to hypercapnia are normal in a comparable group of elite divers studied within 5-6 days of intense apnea training [2]. These results suggest that other factors may account for the absence of increases in MSNA and blood pressure in elite divers.

\section{Are healthy divers protected by absence of co-existing disease/risk factors and/or adaptive responses that oppose chemoreceptor and sympathetic activation?}

Patients with sleep apnea generally have co-existing conditions such as obesity that may promote $\mathrm{CIH}$-induced sympathoexcitation and hypertension [11, 15]. Nonetheless, CIH increases sympathetic nerve activity and blood pressure in healthy young animals [4, 17]. Therefore, absence of disease and risk factors is unlikely to account for the normal function in elite divers.

More likely is the possibility that intense breath-hold diving may engage adaptive mechanisms that oppose the pathological process leading to sustained increases in chemoreflex sensitivity and MSNA. The elite divers in the study by Breskovic et al. [1] exhibited striking respiratory adaptations including increased forced vital capacity, decreased respiratory rate and increased tidal volume. The altered breathing pattern in the divers persisted during the exposure to hypoxia.

Interestingly, breathing pattern can influence autonomic regulation. A voluntary shift to slow deep breathing reduces chemoreflex responses to hypoxia and hypercapnia, improves baroreflex sensitivity, and decreases blood pressure in hypertensive patients $[8,20]$. The mechanisms underlying the favorable responses are unknown, but may involve changes in activity of cardiopulmonary afferent nerves, central respiratory drive and/or psychological state (e.g., anxiety/relaxation). Whether respiratory adaptations in elite divers help maintain normal chemoreflex sensitivity, MSNA and blood pressure-despite a history of $\mathrm{CIH}$-is not known. Of course, beneficial respiratory adaptations as well as other changes in elite divers may arise not only from $\mathrm{CIH}$, but also from other environmental and physical challenges associated with intense breath-hold diving.

Also relevant is the potential for $\mathrm{CIH}$ to improve function. Therapeutic $\mathrm{CIH}$ can induce cardioprotection from myocardial ischemia-reperfusion injury [10], improve autonomic and respiratory control in chronic obstructive pulmonary disease [6], and lower blood pressure in hypertensive humans and animals [18]. Although these benefits are usually achieved with longer exposures to less severe hypoxia than occurs in sleep apnea, the underlying mechanisms may be engaged in elite breath-hold divers.
Can modulation of molecular signaling pathways in carotid body prevent $\mathrm{CIH}$-induced sympathoexcitation?

Molecular mechanisms responsible for $\mathrm{CIH}$-induced chemoreceptor activation are emerging [12, 13, 16, 17]. CIH has two major effects on arterial chemoreceptors; it increases sensitivity to hypoxia, and induces a long-lasting activation of chemoreceptor afferents that persists after return of normal arterial oxygenation [17]. The latter phenomenon can be reproduced in vitro by exposing the carotid body-sinus nerve, isolated from rats or mice previously exposed to $\mathrm{CIH}$ for 10 days, to repetitive cycles of hypoxia/normoxia $[16,17]$. The repetitive hypoxic challenges induce a prolonged increase in chemoreceptor activity referred to as sensory long-term facilitation (sLTF) $[16,17]$.

The mechanisms underlying SLTF in carotid body chemoreceptors involve a host of molecular signaling events. $\mathrm{NAD}(\mathrm{P}) \mathrm{H}$ oxidase-generated reactive oxygen species lead to activation of transcription factors including hypoxiainducible factor-1 (HIF-1), nuclear factor of activated $\mathrm{T}$ cells, and nuclear factor $\kappa \mathrm{B}(\mathrm{NF} \kappa \mathrm{B})[13,16,17]$. Downregulation of HIF-2 and superoxide dismutase-2 further amplify the oxidative stress [12]. Genetic deficiency or pharmacological antagonism of these molecular signaling pathways, or treatment with antioxidants eliminates SLTF and CIH-induced sympathoexcitation [12, 13, 17]. A central role of oxidative stress in CIH-induced chemoreceptor activation is supported by the findings that patients with sleep apnea exhibit oxidative stress [11], and that apneaand exercise-induced oxidative stress is reduced in elite breath-hold divers compared with control subjects [9].

Interestingly, blockade of sLTF does not interfere with acute chemoreceptor sensitivity to hypoxia $[16,17]$. The fact that different mechanisms mediate these two processes opens the door to therapeutic targeting of sustained sympathoexcitation without disrupting the ability to sense acute hypoxia.

\section{Is sympathetic activation inevitable in humans subjected to CIH?}

We return to the original question posed in this editorial. The normal MSNA, blood pressure, and chemoreflex sensitivity in elite breath-hold divers reported by Breskovic et al. [1] and the studies discussed above highlight the potential for adaptive responses and therapeutic targeting of molecular signaling to mitigate $\mathrm{CIH}$-induced chemoreceptor activation. Surely, sympathoexcitation is not inevitable during $\mathrm{CIH}$ ! The challenge is to better understand the mechanisms that mediate deleterious and adaptive responses to the hypoxic insults that accompany disease and 
extreme recreational and vocational activities. Perhaps an understanding of the protection afforded elite breath-hold divers would suggest new strategies for prevention and/or treatment of sleep apnea-induced sympathoexcitation.

\section{References}

1. Breskovic T, Valic Z, Lipp A, Heusser K, Ivancev V, Tank J, Dzamonja G, Jordan J, Shoemaker JK, Eterovic D, Dujic Z (2010) Peripheral chemoreflex regulation of sympathetic vasomotor tone in apnea divers. Clin Auton Res (this issue)

2. Dujic Z, Ivancev V, Heusser K, Dzamonja G, Palada I, Valic Z, Tank J, Obad A, Bakovic D, Diedrich A, Joyner MJ, Jordan J (2008) Central chemoreflex sensitivity and sympathetic neural outflow in elite breath-hold divers. J Appl Physiol 104:205-211

3. Ferretti G (2001) Extreme human breath-hold diving. Eur J Appl Physiol 84:254-271

4. Fletcher EC, Lesske J, Behm R, Miller CC III, Stauss H, Unger T (1992) Carotid chemoreceptors, systemic blood pressure, and chronic episodic hypoxia mimicking sleep apnea. J Appl Physiol 72:1978-1984

5. Guaraldi P, Serra M, Barletta G, Pierangeli G, Terlizzi R, Calandra-Buonaura G, Cialoni D, Cortelli P (2009) Cardiovascular changes during maximal breath-holding in elite divers. Clin Auton Res 19:363-366

6. Haider T, Casucci G, Linser T, Faulhaber M, Gatterer H, Ott G, Linser A, Ehrenbourg I, Tkatchouk E, Burtscher M, Bernardi L (2009) Interval hypoxic training improves autonomic cardiovascular and respiratory control in patients with mild chronic obstructive pulmonary disease. J Hypertens 27:1648-1654

7. Heusser K, Dzamonja G, Tank J, Palada I, Valic Z, Bakovic D, Obad A, Ivancev V, Breskovic T, Diedrich A, Joyner MJ, Luft FC, Jordan J, Dujic Z (2009) Cardiovascular regulation during apnea in elite divers. Hypertension 53:719-724

8. Joseph CN, Porta C, Casucci G, Casiraghi N, Maffeis M, Rossi M, Bernardi L (2005) Slow breathing improves arterial baroreflex sensitivity and decreases blood pressure in essential hypertension. Hypertension 46:714-718

9. Joulia F, Steinberg JG, Wolff F, Gavarry O, Jammes Y (2002) Reduced oxidative stress and blood lactic acidosis in trained breath-hold human divers. Respir Physiol Neurobiol 133:121130

10. Kolar F, Jezkova J, Balkova P, Breh J, Neckar J, Novak F, Novakova $\mathrm{O}$, Tomasova $\mathrm{H}$, Srbova M, Ostadal B, Wilhelm J, Herget J (2007) Role of oxidative stress in PKC- $\delta$ upregulation and cardioprotection induced by chronic intermittent hypoxia. Am J Physiol Heart Circ Physiol 292:H224-H230

11. Lavie L (2009) Oxidative stress-a unifying paradigm in obstructive sleep apnea and comorbidities. Prog Cardiovasc Dis 51:303-312

12. Nanduri J, Wang N, Yuan G, Khan SA, Souvannakitti D, Peng YJ, Kumar GK, Garcia JA, Prabhakar NR (2009) Intermittent hypoxia degrades HIF- $2 \alpha$ via calpains resulting in oxidative stress: implications for recurrent apnea-induced morbidities. PNAS 106:1199-1204

13. Nanduri J, Yuan G, Kumar GK, Semenza GL, Prabhakar NR (2008) Transcriptional responses to intermittent hypoxia. Respir Physiol Neurobiol 164:277-281

14. Narkiewicz K, van de Borne PJ, Pesek CA, Dyken ME, Montano N, Somers VK (1999) Selective potentiation of peripheral chemoreflex sensitivity in obstructive sleep apnea. Circulation 99:1183-1189

15. Peker Y, Hedner J, Norum J, Kraiczi H, Carlson J (2002) Increased incidence of cardiovascular disease in middle-aged men with obstructive sleep apnea: a 7-year follow-up. Am J Respir Crit Care Med 166:159-165

16. Peng Y-J, Nanduri J, Yuan G, Wang N, Deneris E, Pendyala S, Natarajan V, Kumar GK, Prabhakar NR (2009) NADPH oxidase is required for the sensory plasticity of the carotid body by chronic intermittent hypoxia. J Neurosci 29:4903-4910

17. Prabhakar NR, Dick TE, Nanduri J, Kumar GK (2007) Systemic, cellular and molecular analysis of chemoreflex-mediated sympathoexcitation by chronic intermittent hypoxia. Exp Physiol 92.1:39-44

18. Serebrovskaya TV, Manukhina EB, Smith ML, Downey HF, Mallet RT (2008) Intermittent hypoxia: cause of or therapy for systemic hypertension? Exp Biol Med 233:627-650

19. Somers VK, Dyken ME, Clary MP, Abboud FM (1995) Sympathetic neural mechanisms in obstructive sleep apnea. J Clin Invest 96:1897-1904

20. Spicuzza L, Gabutti A, Porta C, Montano N, Bernardi L (2000) Yoga and chemoreflex response to hypoxia and hypercapnia. Lancet 356:1495-1496 